\title{
FKBP5 haplotypes and PTSD modulate the resting-state brain activity in Han Chinese adults who lost their only child
}

Rongfeng Qi (i] ${ }^{1,2}$, Yifeng Luo ${ }^{3}$, Li Zhang ${ }^{4}$, Yifei Weng ${ }^{1}$, Wesley Surento ${ }^{2}$, Neda Jahanshad $\mathbb{D}^{2}$, Qiang Xu', Yan Yin ${ }^{5}$, Lingjiang Li', Zhihong $\mathrm{CaO}^{3}$, Paul M. Thompson ${ }^{2}$ and Guang Ming Lu'

\begin{abstract}
The stress-related gene FKBP5 has been related to dysregulated glucocorticoid receptor (GR) signaling, showing increased GR sensitivity in trauma-exposed subjects with post-traumatic stress disorder (PTSD) but not in those without PTSD. However, the neural mechanism underlying the effects of FKBP5 remains poorly understood. Two hundred and thirty-seven Han Chinese adults who had lost their only child were included. Four FKBP5 single nucleotide polymorphisms (rs3800373, rs9296158, rs1360780, and rs9470080) were genotyped. All 179 participants were successfully divided into three FKBP5 diplotype subgroups according to two major FKBP5 $\mathrm{H} 1$ and $\mathrm{H} 2$ yin yang haplotypes. Brain average spectral power was compared using a two-way (PTSD diagnosis and FKBP5 diplotypes) analysis of covariance within four separate frequency bands (slow-5, slow-4, slow-3, and slow-2). Adults with PTSD showed lower spectral power in bilateral parietal lobules in slow-4 and in left inferior frontal gyrus (IFG) in slow-5. There was significant FKBP5 diplotype main effect in anterior cingulate cortex (ACC) in slow-4 (H1/H1 higher than other two subgroups), and in precentral/postcentral gyri and middle cingulate cortex (MCC) in slow-3 (H2/H2 higher than other two subgroups). Also, there was a significant diagnosis $\times$ FKBP5 diplotype interaction effect in right parietal lobule in slow-3. These findings suggest that adults with PTSD have lower low-frequency power in executive control network regions. Lower power in ACC and greater power in the motor/sensory areas in FKBP5 high-risk diplotype group suggest a disturbance of emotional processing and hypervigilance/sensitization to threatening stimuli. The interaction effect of diagnosis $\times$ FKBP5 in parietal lobule may contribute to PTSD development.
\end{abstract}

\section{Introduction}

Post-traumatic stress disorder (PTSD) is characterized by dysregulation of the hypothalamus-pituitary-adrenal (HPA) axis after exposure to traumatic events, displayed as enhanced negative-feedback inhibition of the HPA axis $^{1,2}$. One typical clinical feature of this abnormality in

\footnotetext{
Correspondence: Paul M. Thompson (pthomp@usc.edu) or Guang Ming Lu (cjr.luguangming@vip.163.com)

'Department of Medical Imaging, Jinling Hospital, Medical School of Nanjing University, 210002 Nanjing, Jiangsu, China

${ }^{2}$ Imaging Genetics Center, Mark and Mary Stevens Neuroimaging and Informatics Institute, University of Southern California, Marina del Rey, CA 90292, USA

Full list of author information is available at the end of the article

These authors contributed equally: Rongfeng Qi, Yifeng Luo
}

PTSD is an exaggerated suppression of cortisol in response to dexamethasone administration and the enhanced sensitivity of glucocorticoid receptors $(\mathrm{GR})^{3,4}$. Even so, only a minority of trauma-exposed individuals will eventually develop PTSD ${ }^{5}$, and the increased GR sensitivity following stress happens only in PTSD but not in non-PTSD subjects ${ }^{3,4}$. The underlying mechanisms of the association between PTSD development and GR sensitivity remain poorly understood.

Genetic factors that regulate GR signaling influence the individual differences in the HPA axis response to stress $^{6,7}$. A critical regulatory gene is the FK506-binding protein 5 (FKBP5) gene which encodes the FKBP5 protein $^{8}$. In the absence of cortisol, FKBP5 binds to the GR 
complex, resulting in decreased affinity for cortisol and less efficient nuclear translocation of GR. In the presence of cortisol, FKBP5 is exchanged with other co-chaperones, causing more efficient translocation of GR to the nucleus ${ }^{9}$. Healthy carriers of specific FKBP5 single nucleotide polymorphisms (SNPs) were tended to have higher FKBP5 protein expression and thus decreased cortisol affinity and nuclear translocation of GR, resulting in GR resistance ${ }^{6}$, suggesting the presence of protective features after response to stress. However, this functional association is switched in PTSD $^{6}$, in which the same FKBP5 SNPs were associated with increased GR sensitivity, conferring "risk" SNPs for PTSD. So far, little is known about the exact mechanisms of different modulatory effects of FKBP5 risk SNPs in people who experienced traumatic events with and without PTSD.

Imaging genomics is significantly improving our understanding of human brain function, and offers a means to study molecular and cellular abnormalities that are implicated in many psychiatric disorders in which genes play an important role ${ }^{10-13}$. To the best of our knowledge, only one imaging genomics study with a small sample size (a total of 54 subjects) has investigated the effect of FKBP5 risk SNP polymorphisms on brain function of $\mathrm{PTSD}^{14}$. In that study, Fani et al. firstly reported that PTSD patients who carried two FKBP5 rs1360780 risk SNPs had lower fractional anisotropy in the cingulum, compared to other diagnostic and genotype groups. Also, trauma-exposed subjects who carried two FKBP5 rs1360780 risk SNPs had lower functional connectivity of hippocampus-anterior cingulate cortex than non-risk SNP carriers, regardless of PTSD diagnosis ${ }^{14}$. The FKBP5 rs1360780 has been demonstrated to function together with other FKBP5 SNPs which are in strong linkage disequilibrium, resulting in gene dose-dependent regulation on the HPA axis ${ }^{6}$. Several studies have focused on the two major functional FKBP5 haplotypes driven from four SNPs (rs3800373, rs9296158, rs1360780, and rs9470080)-the H1 (carrying zero risk SNPs: AGCC) and H2 (carrying four risk SNPs: CATT) yin yang haplotypes ${ }^{15,16}$. However, no imaging genomics study to date has used this desirable haplotype to investigate the modulatory effects of FKBP5 on brain function in PTSD development. In a recent fMRI study examining effects of FKBP5 risk SNPs, Bryant et al. divided the healthy participants into high-risk and low-risk genogroups based on the number of risk alleles carried (using the abovementioned four FKBP5 SNPs) and then investigated the differences in resting-state brain function between these two genogroups ${ }^{17}$. They found that the high-risk genogroup showed less power in low frequency range but more power in higher frequency range in the frontotemporoparietal network, compared to the low-risk genogroup.
In this prospective study, we aimed to examine the modulatory effects of $F K B P 5 \mathrm{H} 1$ and $\mathrm{H} 2$ yin yang haplotypes on resting state brain function using resting-state fMRI in trauma-exposed subjects with and without PTSD. A wide variation in the types of trauma and differences in race/ethnicity of the populations studied might potentially influence the findings of imaging genomics analyses ${ }^{18}$. Therefore, we conducted a PTSD survey in a specific group of Han Chinese adults who had lost their only child. By doing so, we restricted our study to participants of the same ethnicity (Han Chinese) and homogenous traumatic event (loss of only child). Power spectrum analysis ${ }^{19,20}$ an important fMRI approach that measures the strength of intrinsic brain activity-was used in this study for three reasons. Firstly, the power spectrum, especially within the low-frequency band, is a physiologically meaningful and powerful biomarker in exploring human spontaneous brain activity ${ }^{20-22}$, and has been successfully applied in prior genetic association studies in several mental and neuropsychiatric disorders such as mild cognitive impairment ${ }^{23,24}$ and major depressive disorder ${ }^{25}$. Secondly, in a prior imaging genomics study using four FKBP5 risk SNPs, only spectral power demonstrated significant differences between the high-risk and low-risk genogroups ${ }^{17}$. Thirdly, the power spectrum analysis is a whole-brain algorithm that needs no region-specific hypothesis, which might be more suitable for a preliminary study. We hypothesized that the FKBP5 haplotypes would exhibit different modulatory effects on brain spectral power in Han Chinese adults who had lost their only child with and without PTSD.

\section{Methods \\ Subjects}

The present study was approved by the Medical Research Ethics Committee of Jiangsu University. Each participant provided written informed consent. Between September 2016 and March 2017, we conducted a survey in Jiangsu Province, China on a population of the Han Chinese adults who had lost their only child during the period of "One-Child Policy"26,27. All 237 Han adults who had lost their only child-without other major traumatic exposures based on the clinician-administered PTSD scale (CAPS) life events checklist-were successfully interviewed and screened by the clinician-administered PTSD scale (CAPS). They were also further screened with the Chinese version of the structured clinical interview for DSM-IV (SCID) ${ }^{28}$ which was revised by Prof. Lipeng Fei from the Beijing Hui Long Guan Hospital. After doing this, 170 out of the total of 237 adults did not meet any diagnostic criteria for mental illness (including current or lifetime PTSD) or substance use disorders; 57 adults were diagnosed with PTSD; the remaining 10 adults were diagnosed with other psychiatric disorders ( 5 with major 
depressive disorder, 4 with generalized anxiety disorder, and 1 with both depression and anxiety diagnosis) and they were not included in the current study.

Exclusion criteria for the following MRI study were as follows: any current or history of brain injury or other major medical or neurological conditions (4 adults without PTSD were excluded for cerebral infarction or ischemia, and 1 adult without PTSD was ruled out for a history of major depressive disorder and antidepressant drug therapy); any MRI contraindication (none); and lefthandedness (none).

\section{MR scanning}

MR imaging was performed with a 3-T scanner (Achieva 3.0 TTX; Philips, Amsterdam, the Netherlands). Each participant was instructed to stay still in the scanner during image acquisition, keep eyes closed, remain awake and move as little as possible. $\mathrm{T}_{1}$-weighted anatomical images were acquired using a three-dimensional turbo fast echo sequence (repetition time ms/echo time msec [TR/TE], 9.7/4.6; flip angle, $9^{\circ}$; field of view (FOV), $256 \times$ $256 \mathrm{~mm}^{2}$; matrix size, $256 \times 256$; slice thickness, $1 \mathrm{~mm}$; 160 sagittal slices). For resting-state functional imaging, we performed a single-shot, gradient-recalled echo-planar imaging sequence (TR/TE, 2000/30; flip angle, $90^{\circ}$; FOV = $192 \times 192 \mathrm{~mm}^{2}$; matrix, $64 \times 64$; voxel size, $3 \times 3 \times 4 \mathrm{~mm}^{3}$; volume number, 230; 35 axial slices). The total scan time for resting-state functional imaging was $460 \mathrm{~s}$.

\section{Measures}

All participants were assessed with neuropsychological tests, which included: the Hamilton Depression $(\text { HAMD })^{29}$ and Hamilton Anxiety (HAMA) ${ }^{30}$ rating scales, Mini-Mental State Examination (MMSE) ${ }^{31}$, Chinese Social Support Rating Scale (SSRS) with sections about subjective support, objective support and the utility of support ${ }^{32}$, and individual Simple Coping Style Questionnaire (SCSQ) with active and negative coping score, and the coping tendency score ${ }^{33}$. A detailed description is available in the online Supplementary Note 1.

\section{Data preprocessing}

MRI data was preprocessed with the Data Processing Assistant for Resting-State fMRI (DPARSF, http://rfmri. org/DPARSF) which is based on Statistical Parametric Mapping (http://www.fil.ion.ucl.ac.uk/spm). First, the initial ten volumes were excluded. Then, the slice timing and head motion correction were conducted on all the remaining volumes. Individual $\mathrm{T}_{1}$-weighted images were co-registered to the functional images and then segmented into gray matter, white matter, and cerebrospinal fluid, and transformed into the standard Montreal Neurological Institute (MNI) space using the Diffeomorphic Anatomical Registration Through Exponentiated Lie algebra (DARTEL) method ${ }^{34}$. Finally, the functional images were transformed into the MNI stereotaxic space $\left(3 \times 3 \times 3 \mathrm{~mm}^{3}\right)$, using the parameters of the $\mathrm{T}_{1}$-weighted image normalization, and then smoothed with an $8 \mathrm{~mm}$ full width at half maximum (FWHM) isotropic Gaussian kernel.

\section{Quality control and nuisance regression}

To minimize head motion confounds, we used the Friston 24-parameter model ${ }^{35}$ to regress out head motion effects. Individual head translations, rotations, and framewise displacement (using the Jenkinson formula) were calculated. Seven adults were excluded (5 without PTSD, and 2 with PTSD) for head translations $>1.5 \mathrm{~mm}$ or rotations $>1.5^{\circ}$, and 2 adults (without PTSD) were excluded for mean framewise displacement $>2.5$ standard deviations. The mean framewise displacement of each participant was also included as a nuisance covariate in the statistical analysis of the fMRI data ${ }^{36,37}$. Mean signals from cerebrospinal fluid and white matter were also regressed out as spurious variance to restrict the analysis to gray matter.

After performing the quality control, a total of nine participants were excluded, and 55 adults with PTSD and 158 adults without PTSD remained.

\section{Power spectrum analysis}

Firstly, for a given voxel, the time course was converted to the frequency domain using a fast Fourier transform $^{20,22}$. Then, the square root of the power spectrum was computed and averaged across a predefined frequency interval. Based on the most current knowledge, some brain disorders ${ }^{38,39}$, as well as some genes ${ }^{17}$, may selectively affect the brain power spectrum within a certain frequency band. Here, we have divided the full frequency range $(0-0.25 \mathrm{~Hz})$ into four narrowly-defined bands according to prior studies ${ }^{21,22}$ : slow-5 $(0.01-0.027 \mathrm{~Hz}), \quad$ slow- $4 \quad(0.027-0.073 \mathrm{~Hz}), \quad$ slow-3 $(0.073-0.198 \mathrm{~Hz})$, and slow-2 $(0.198-0.25 \mathrm{~Hz})$; and we calculated the average power spectrum in individual frequency band. For standardization purposes, the average power spectrum of each voxel was divided by the global mean values.

\section{DNA genotyping}

With the exception of three adults without PTSD who refused the blood collection procedure, we successfully collected DNA data from all other participants from peripheral blood samples. Four FKBP5 SNPs (rs3800373, rs9296158, rs1360780, and rs9470080) were genotyped using the Improved Multiple Ligase Detection Reaction (iMLDR) technique developed by Genesky Biotechnologies, Inc. (Shanghai, China) ${ }^{40}$. Detailed primers for these four SNPs of FKBP5 are listed in Supplementary Table 1. 


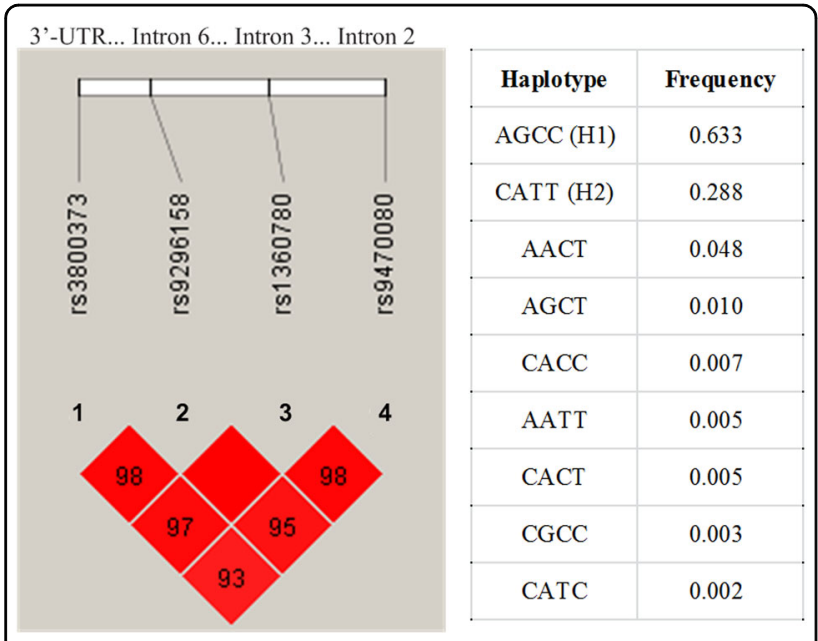

Fig. 1 The FKBP5 4-SNP haplotype block structure, and two major H1 (yin) $\mathbf{H} 2$ (yang) functional haplotypes. The FKBP5 $\mathrm{H} 1$ (yin) haplotype carries zero risk SNPS (AGCC); the H2 (yang) haplotype carries all four risk SNPs (CATT). $\mathrm{H} 1$ and $\mathrm{H} 2$ haplotypes account for $92.1 \%$ of the haplotype diversity in this study. SNP single-nucleotide polymorphism, UTR untranslated regions.

About 5\% of the samples were randomly selected for confirmation, and the results were $100 \%$ concordant. For all 210 study participants with available gene data-and who passed quality control for their fMRI data-the distributions of rs3800373, rs9296158, rs1360780, and rs9470080 did not differ from Hardy-Weinberg equilibrium ( $P=0.74,0.45,0.62$, and 0.30 , respectively) calculated using $\mathrm{R}$, version 3.5.3 (https://www.r-project.org).

\section{FKBP5 haplotype estimation}

A haplotype is a combination of alleles that are located close together on the same chromosome and inherited together ${ }^{41}$. Here, we used Haploview version $4.2^{42}$ and genotype data to compute the linkage disequilibrium blocks for these four FKBP5 SNPs, and found they were in strong linkage disequilibrium (Fig. 1) and in approximate allelic identity (Supplementary Table 2). There were 9 FKBP5 haplotypes (frequency $\geq 0.01$ ) identified in this study, with two major functional haplotypes-the $\mathrm{H} 1$ and $\mathrm{H} 2$ yin yang haplotypes (Fig. 1). The H1 (yin) haplotype carries zero risk SNPs (AGCC), while the H2 (yang) haplotype carries all four risk SNPs $(\mathrm{CATT})^{6,15,16}$. These two major functional haplotypes accounted for $92.1 \%$ of haplotype diversity in this study, which is similar to findings in prior studies ${ }^{15,16}$. Then, PHASE version $2.1^{43-45}$ was performed to determine the most probable haplotype assignments for each individual by assessing the probability of each possible haplotype and determining a confidence score. A diplotype represents a pair of haplotypes on homologous chromosomes and could provide more complete genetic information ${ }^{46,47}$. For this reason, the FKBP5 diplotype was further estimated for 210 study participants; 179 of them carried homozygote and heterozygote combinations of the $\mathrm{H} 1$ and $\mathrm{H} 2$ yin yang haplotypes and were included in the final diplotype based neuroimaging analysis. From these 179 adults, 87 were $\mathrm{H} 1 / \mathrm{H} 1$ (64 without PTSD, 23 with PTSD), 72 were $\mathrm{H} 1 / \mathrm{H} 2$ (51 without PTSD, 21 with PTSD), and 20 were H2/H2 (15 without PTSD, 5 with PTSD) (Supplementary Table 3$)$.

\section{Statistical analysis}

SPSS version 25 (IBM Corp, Armonk, New York, USA) was used to analyze the demographic and neuropsychological data. A two-way (diagnosis of PTSD, FKBP5 diplotypes) analysis of variance was used to evaluate the main effects of diagnosis, FKBP5 diplotypes and their interaction effects on demographic and neuropsychological data. A voxel-wise two-way (diagnosis, FKBP5 diplotypes) full factorial analysis of covariance (ANCOVA) was performed using SPM12 to assess the main effect of PTSD diagnosis, FKBP5 diplotypes and their interaction effects on brain power spectrum maps within the four different frequency bands, adjusting for effects of age, sex, educational level, duration since childloss trauma, and framewise displacement due to head motion. The frequency band was not considered as a repeated factor in this paper as we wanted to primarily focus on the effects that diagnosis and genetic variation may exert on the power spectrum within different bands, but not the differences among different bands themselves. Results were corrected for multiple comparisons (corrected $P<0.05)$ using random-field theory (RFT) ${ }^{48}$ with initial voxel level $P<0.001$ and cluster level $P<0.05$.

A partial correlation analysis was performed to examine the relationship between regions with significantly different power spectra and CAPS, SSRS, SCSQ, HAMA, and HAMD, with the inclusion of age, sex, educational level, duration since child-loss trauma, and head motion as covariates. Correlation results were corrected for multiple comparisons using the Bonferroni correction for the number of regions where altered power spectra were detected from the two-way ANCOVA (cut-off $P$ values of $0.05 / 8=0.006$, corresponding to all eight regions showing differences in this study).

\section{Results}

\section{Clinical and neuropsychological data}

A flowchart describing the study population is detailed in Supplementary Fig. 1. All 179 adults (49 with PTSD and 130 without PTSD) who carried homozygote and heterozygote combinations of the $\mathrm{H} 1$ (yin) and $\mathrm{H} 2$ (yang) haplotypes were included in the final FKBP5 diplotypes based neuroimaging analyses (Table 1 and Supplementary Table 3). 
Table 1 Demographics and neuropsychological data of Han Chinese adults who lost and only child and carried heterozygous/homozygous combinations of 2 major FKBP5 haplotypes.

\begin{tabular}{llll}
\hline Protocols & $\begin{array}{l}\text { Adults with } \\
\text { PTSD }(\boldsymbol{n}=\mathbf{4 9})\end{array}$ & $\begin{array}{l}\text { Adults without } \\
\text { PTSD }(\boldsymbol{n}=\mathbf{1 3 0})\end{array}$ & $\boldsymbol{P}$ value \\
\hline Age ( \pm SD) (years) & $57.76 \pm 5.59$ & $58.79 \pm 5.46$ & $0.26^{\mathrm{a}}$ \\
Sex (F/M) & $35 / 14$ & $60 / 70$ & $0.003^{\mathrm{b}}$ \\
Education (years) & $6.43 \pm 4.24$ & $6.58 \pm 3.58$ & $0.82^{\mathrm{a}}$ \\
HAMD & $15.84 \pm 6.77$ & $5.93 \pm 4.19$ & $<0.001^{\mathrm{a}}$ \\
HAMA & $12.65 \pm 6.71$ & $4.57 \pm 3.41$ & $<0.001^{\mathrm{a}}$ \\
MMSE & $25.69 \pm 3.17$ & $26.13 \pm 3.44$ & $0.44^{\mathrm{a}}$ \\
Duration since child-loss & $59.71 \pm 49.82$ & $107.09 \pm 72.39$ & $0.001^{\mathrm{a}}$ \\
trauma, month & & & \\
CAPS_total & $46.69 \pm 12.66$ & $16.35 \pm 9.95$ & $<0.001^{\mathrm{a}}$ \\
SSRS & & & \\
Objective support & $12.27 \pm 2.77$ & $12.64 \pm 2.75$ & $0.42^{\mathrm{a}}$ \\
Subjective support & $21.55 \pm 3.82$ & $21.47 \pm 3.89$ & $0.90^{\mathrm{a}}$ \\
Utility of support & $5.63 \pm 2.07$ & $5.48 \pm 1.94$ & $0.65^{\mathrm{a}}$ \\
SSRS_total & $39.45 \pm 7.13$ & $39.58 \pm 6.64$ & $0.91^{\mathrm{a}}$ \\
SCSQ & & & $0.31^{\mathrm{a}}$ \\
Active & $18.27 \pm 6.35$ & $19.38 \pm 6.46$ & $0.38^{\mathrm{a}}$ \\
Negative & $10.08 \pm 3.04$ & $10.37 \pm 3.34$ & $9.05 \pm 6.01$ \\
Copying tendency & $8.18 \pm 5.70$ & & \\
\hline
\end{tabular}

PTSD post-traumatic stress disorder, HAMD Hamilton depression, HAMA Hamilton anxiety, MMSE mini-mental state examination, CAPS clinicianadministered PTSD scale, SSRS social support rating scale, SCSQ simple coping style questionnaire.

Values are expressed as mean \pm SD.

${ }^{\text {a }}$ The $P$ value for the difference between the two trauma-exposed groups was obtained by two-sample $t$-test.

${ }^{b}$ The $P$ value for gender distribution between the two trauma-exposed groups was obtained by the chi-square test.

There were no significant differences between PTSD and non-PTSD groups in age, educational level, SSRS, or SCSQ (all $P>0.05$ ), but the PTSD group showed higher CAPS, HAMA and HAMD scores, higher male/female ratio, and shorter duration since losing the child (Table 1) than the non-PTSD groups. There was no significant diagnosis or FKBP5 diplotypes main effect, and no interaction effect on other clinical and neuropsychological data.

\section{The main effect of PTSD diagnosis}

Significant PTSD diagnosis effects on spectral power were found in bilateral parietal lobules in slow-4 band and in left inferior frontal gyrus (IFG) in slow-5 band. Posthoc analysis showed that PTSD adults had lower spectral power in these regions, relative to trauma-exposed adults without PTSD (Fig. 2 and Supplementary Table 4).

\section{The main effect of $F K B P 5$ diplotypes}

There was significant $F K B P 5$ diplotypes main effect on spectral power in the anterior cingulate cortex (ACC) in slow-4 band, and in the bilateral precentral/postcentral gyri and middle cingulate cortex (MCC) in slow-3 band in all participants, irrespective of PTSD diagnosis (Fig. 3 and Supplementary Table 4). At ACC, the H1/H1 diplotype subgroup had higher spectral power than other two genogroups. For the precentral/postcentral gyri and $\mathrm{MCC}$, the $\mathrm{H} 2 / \mathrm{H} 2$ diplotype group had higher spectral power than other two genogroups. The FKBP5 diplotype modulation accounted for $14 \%$ of the variance in spectral power in left precentral/postcentral gyri, $10 \%$ in right precentral/postcentral gyri, $10 \%$ in the right MCC and $9 \%$ in the ACC (partial eta squared $\left[\eta^{2}\right]=14,10,10$, and $9 \%$, respectively).

\section{Interaction of FKBP5 diplotypes and PTSD diagnosis}

There was a significant diagnosis $\times$ FKBP5 diplotype interaction effect on spectral power in right parietal lobule in slow-3 band (Fig. 4 and Supplementary Table 4). Post-hoc analysis showed that within non-PTSD adults, the $\mathrm{H} 2 / \mathrm{H} 2$ diplotype subgroup had higher spectral power than other two genogroups; whereas within PTSD adults, the $\mathrm{H} 2 / \mathrm{H} 2$ diplotype subgroup had lower spectral power than the other two genogroups (Fig. 4). This diagnosis $x$ FKBP5 diplotype interaction accounted for $10 \%$ of the variance in spectral power in right parietal lobule $\left(\eta^{2}=10 \%\right)$.

\section{Partial correlation results}

A marginally negative partial correlation was found between the power spectrum in right parietal lobule in slow-4 band and CAPS scores, albeit only in the PTSD group ( $r=-0.27 ; P=0.06$, Supplementary Fig. 2). Power spectra in brain regions affected by FKBP5 diplotypes and diagnosis $\times F K B P 5$ interaction were not correlated with any clinical or neuropsychological indices.

\section{Additional analyses of structural data}

To evaluate whether the functional results in this study might be confounded by brain structural changes, we further performed voxel-based morphometry (VBM) to examine possible structural deficits, using the CAT12 Toolbox (http://dbm.neuro.uni-jena.de/cat12/). We used the default settings detailed in the manual for CAT12 (http://dbm.neuro.uni-jena.de/cat12/CAT12-Manual.pdf) except for applying the affine regularization using the International Consortium for Brain Mapping template for East Asian brains. The individual $\mathrm{T}_{1}$-weighted images were finally segmented into gray matter, white matter and cerebrospinal fluid. The segmented gray matter images were smoothed with an FWHM of $8 \mathrm{~mm}$. Regional gray matter volume of each region showing significant 


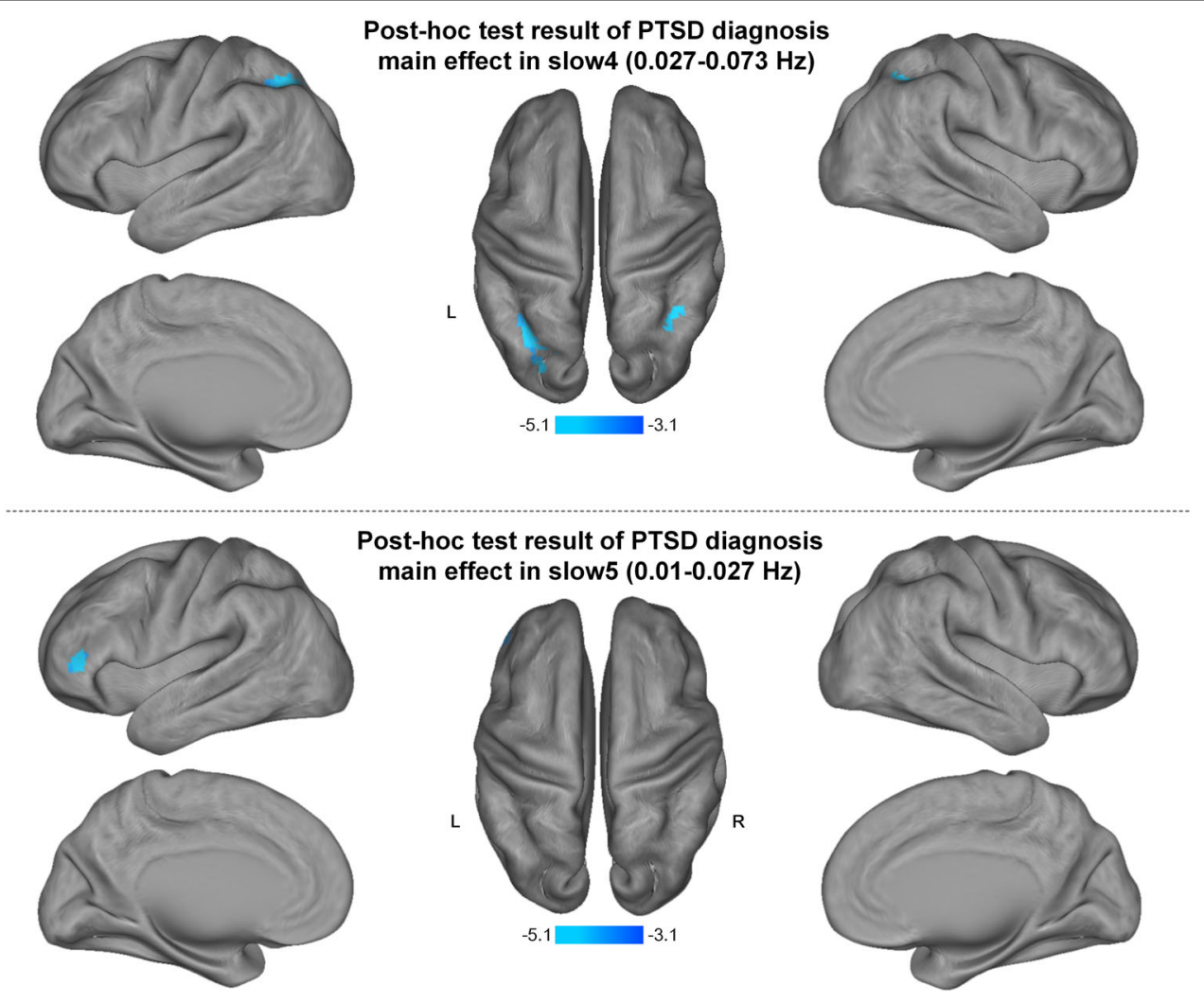

Fig. 2 Post-hoc test result of PTSD diagnosis main effect on brain resting-state power spectrum (corrected $P<0.05$ ). POSt-hoc analysis of PTSD main effect shows that PTSD adults have lower spectral power in bilateral parietal lobules in slow- 4 and in left inferior frontal gyrus in slow- 5 band, relative to trauma-exposed adults without PTSD.

differences in FKBP5 diplotypes based neuroimaging analyses was extracted and then compared with a similar two-way ANCOVA. No significant diagnosis or FKBP5 main effect or their interaction effect on gray matter volume was found in these regions.

\section{Discussion}

In this study, we investigated the effects of PTSD diagnosis and stress-related gene FKBP5 on spontaneous brain activity in Han Chinese adults who had lost their only child. We found a main effect of PTSD diagnosis on low-frequency power (slow- 4 and slow- 5 bands) in the parietal lobules and inferior frontal gyrus, and a main effect of FKBP5 diplotype on low-frequency power (slow4 band) in ACC and middle-frequency power (slow-3 band) in the motor/sensory areas irrespective of PTSD diagnosis. Also, there was a significant diagnosis $\times F K B P 5$ interaction effect on middle-frequency power (slow-3 band) in parietal lobule.

The prominent neuroanatomical theory of PTSD points to a decreased prefrontal inhibitory control over the amygdala ${ }^{49}$, supported by quantitative neuroimaging studies $^{50,51}$ and meta-analytic reviews ${ }^{49,52}$. Other evidence also supports the cognitive-affective imbalance theory in the pathology of $\mathrm{PTSD}^{53,54}$, as evidenced by underactivated regions within the brain executive system and overactivated regions within the emotional processing system. The parietal lobule is a core component of the brain executive control network ${ }^{55,56}$. Decreased parietal lobule activity has been demonstrated in adults with a history of early life stress exposure ${ }^{57,58}$ and patients with PTSD $^{54,59}$. Its activity has also been found to be negatively correlated with PTSD symptoms ${ }^{54}$. Both right ${ }^{60}$ and left ${ }^{61}$ inferior frontal gyri play an important role in attentional monitoring and inhibiting inappropriate responses. Reduced inferior frontal gyrus activity during a proactive inhibition task was reported in veterans with PTSD, relative to control veterans without $\mathrm{PTSD}^{62}$. Thus, in this study, the lower low-frequency power in parietal lobule and inferior frontal gyrus aligns with the findings in prior studies about PTSD. The marginally negative correlation between parietal lobule and CAPS in PTSD adults in this study also supports our findings.

Twin studies posit that PTSD is moderately heritable, with heritability estimates in the range of $28-46 \%{ }^{63-65}$. Increased GR sensitivity is only found in trauma-exposed subjects with PTSD rather than those without PTSD ${ }^{6}$. As a critical modulator of GR sensitivity, FKBP5 is thought to 


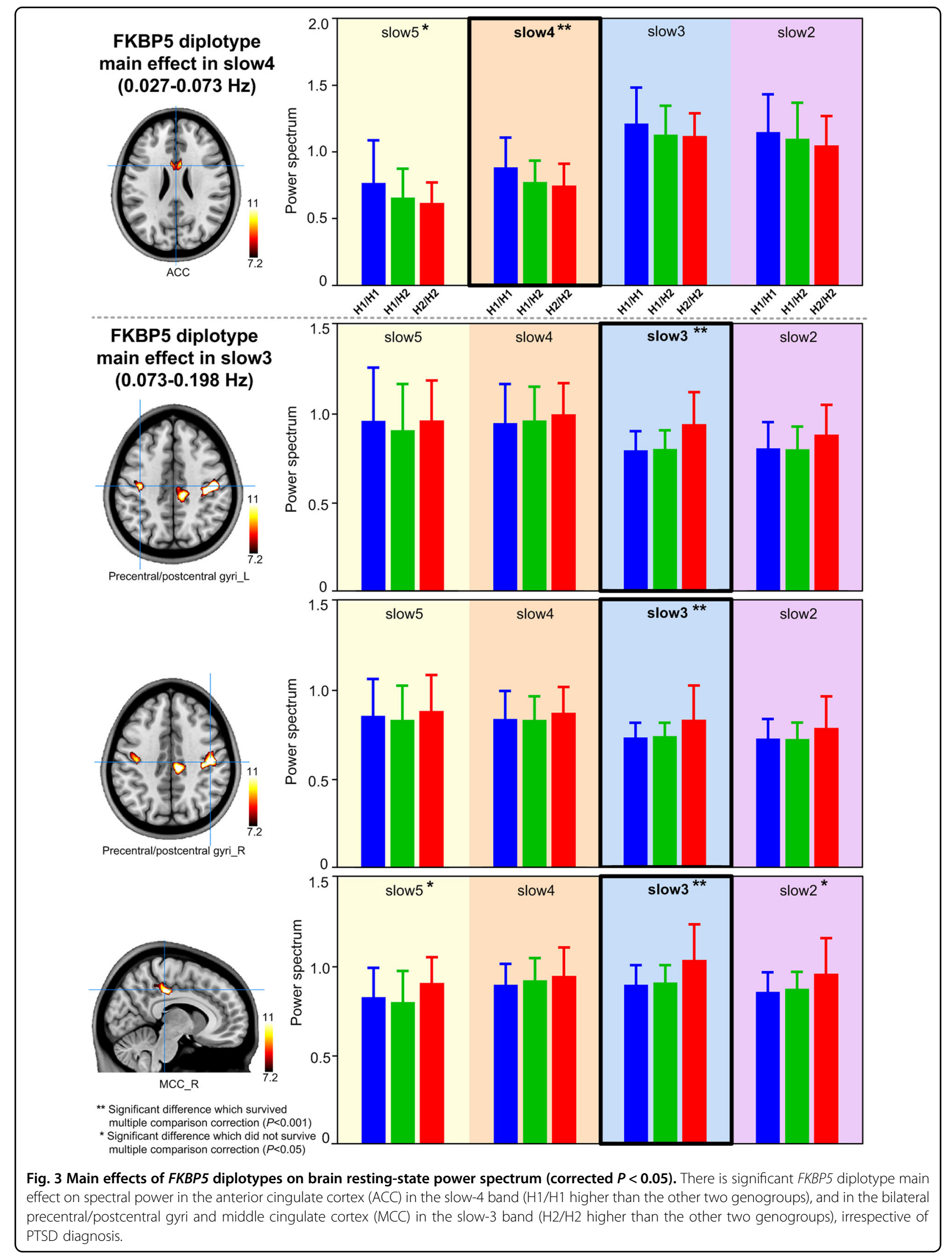




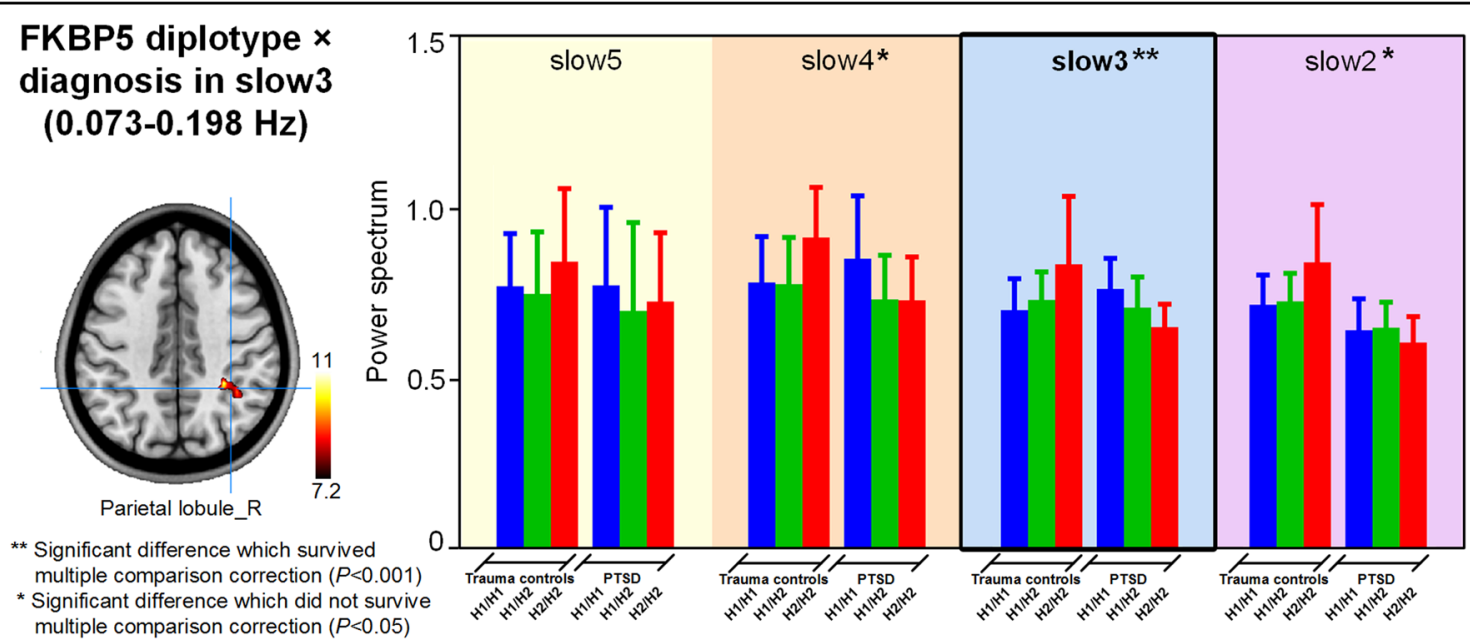

Fig. 4 Interaction effect of diagnosis $\times$ FKBP5 diplotypes effect on brain resting-state power spectrum (corrected $P<0.05$ ). There is a significant diagnosis $\times$ FKBP5 diplotype interaction effect on spectral power in right parietal lobule in slow-3 band. Within non-PTSD adults, the H2/ $\mathrm{H} 2$ diplotype subgroup has higher spectral power than the other two genogroups; whereas within PTSD adults, the H2/H2 diplotype subgroup has lower spectral power than the other two genogroups.

be an interesting candidate gene for PTSD development ${ }^{66}$. Individuals with PTSD who carried two FKBP5 rs1360780 risk SNPs (TT) exhibited the lowest cingulum fractional anisotropy, compared to the same risk SNPs carriers without PTSD, and other genotypes both with and without PTSD ${ }^{14}$. The FKBP5 diplotypes with homozygote and heterozygote combinations of the $\mathrm{H} 1$ and $\mathrm{H} 2$ yin yang haplotypes provide more complete genetic information $^{46,47}$. A recent study divided a group of healthy participants into high and low-risk genogroups according to the number of risk alleles of four FKBP5 SNPs, and found that the FKBP5 high-risk allele group demonstrated lower low-frequency power spectrum but greater highfrequency power spectrum in brain frontotemporoparietal network, compared to the low-risk allele group ${ }^{17}$. To the best of our knowledge, our current study is the first to further describe the effect of FKBP5 diplotypes on restingstate brain activity in trauma-exposed subjects both with and without PTSD diagnosis. In this study, at ACC, the zero-risk diplotype group $(\mathrm{H} 1 / \mathrm{H} 1)$ displayed higher lowfrequency power spectrum than the middle $(\mathrm{H} 1 / \mathrm{H} 2)$ - and high $(\mathrm{H} 2 / \mathrm{H} 2)$ - risk diplotype groups; while at precentral/ postcentral gyri and $\mathrm{MCC}$, the $\mathrm{H} 2 / \mathrm{H} 2$ diplotype group had higher middle spectral power than other two genogroups. Although using different study samples and different FKBP5 gene grouping methods, both our current results and findings from prior study ${ }^{17}$ suggest that the FKBP5 risk alleles may have different modulatory effects on brain activity within different frequency bands, where high-risk alleles may associate with lower low-frequency power but greater middle or high-frequency power in frontotemporoparietal regions. In the $F K B P 5$ high-risk genogroup, the lower power spectrum in ACC may indicate impaired emotional processing, while the higher power spectrum in precentral/postcentral gyri may suggest hypervigilance/sensitization to threatening stimuli ${ }^{17}$. This interpretation is partially supported by a prior study on FKBP5 rs1360780 in subjects recruited from the general medical clinics, where risk allele (TC/TT) carriers showed attention bias toward the threat, compared to non-risk allele carriers $(C C)^{67}$.

In the present study, another important finding is the interaction modulatory effect of FKBP5 $\times$ diagnosis in the parietal lobule, where in PTSD adults, the high-risk diplotype group was associated with lower spectral power than the other two genogroups, whereas, in non-PTSD adults the opposite occurred. One possible interpretation for the different modulatory effects of FKBP5 risk diplotype in PTSD and non-PTSD subjects is that some brain regions-such as the parietal lobule-may have a resilient or compensatory role in trauma-exposed subjects without PTSD. Further research is needed to gain a precise understanding of the role of FKBP5 risk diplotype on PTSD development. For example, more quantitative neuroimaging-genetics studies are required to provide reliable and repeatable intermediate phenotypes that would improve our understanding of GR sensitivity differences in subjects with PTSD and without PTSD.

This study has several limitations. First, our study only focused on the influence of losing an only child in China, and so we urge caution when applying these results to other traumatic experiences and other populations of different race or ethnicity. Second, due to the crosssectional nature of the current study, it was unclear if the brain differences were present before the traumatic experience or if they occurred after the traumatic event. 
Third, an increasing number of studies have demonstrated that the FKBP5 risk SNPs often interact with individual childhood trauma, resulting in an increased risk of PTSD development and greater PTSD symptoms ${ }^{66,68}$. This evidence suggests the importance of gene $\times$ childhood environment interactions for PTSD. However, childhood trauma data was not originally collected in this study and thus needs to be taken into account in further studies. Finally, given the significant history of failure to replicate candidate-gene studies ${ }^{69,70}$, the findings here should be considered as preliminary results and need to be validated by studies involving large replication samples or using data from genome-wide association studies (GWAS) of PTSD.

\section{Conclusion}

In conclusion, our findings suggest that PTSD may impair lower low-frequency power in executive control network regions in Han Chinese adults who had lost their only child. The FKBP5 high-risk diplotype group displayed lower spectral power in ACC and greater power in the motor/sensory areas, suggesting a disturbance of emotional processing and hypervigilance/sensitization to threatening stimuli. The interaction effect of diagnosis $x$ FKBP5 in parietal lobule may contribute to PTSD development in adults who experienced the loss of an only child.

\section{Acknowledgements}

This work was funded by the grants from the National Nature Science Foundation of China [Nos. 81671672, 81301209 to R.Q., and 81801678 to Y.L.]; the Jiangsu Provincial Medical Youth Talent [Nos. QNRC2016888 to R.Q., and QNRC2016207 to Y.L.]; the Chinese Key Grant [No. BWS11J063 to G.M.L.]; the Youth Nature Science Foundation of Jiangsu Province [No. BK20170223 to Y.L.]; and NIH [No. U54 EB020403 to P.M.T. and N.J.].

\begin{abstract}
Author details
'Department of Medical Imaging, Jinling Hospital, Medical School of Nanjing University, 210002 Nanjing, Jiangsu, China. ${ }^{2}$ Imaging Genetics Center, Mark and Mary Stevens Neuroimaging and Informatics Institute, University of Southern California, Marina del Rey, CA 90292, USA. ${ }^{3}$ Department of Radiology, The Affiliated Yixing Hospital of Jiangsu University, 75 Tongzhenguan Road, 214200 Wuxi, China. ${ }^{4}$ Mental Health Institute of the Second Xiangya Hospital, Central South University, China National Clinical Research Center for Mental Health Disorders, National Technology Institute of Psychiatry, 139 Middle Renmin Road, 410011 Changsha, Hunan, China. ${ }^{5}$ sychology Department, Hangzhou Seventh People's Hospital, 310013 Hangzhou, Zhejiang, China
\end{abstract}

\section{Conflict of interest}

All authors declare no competing interests. P.M.T. and N.J. received a research grant from Biogen, Inc., (Boston, USA), for research unrelated to this manuscript.

\section{Publisher's note}

Springer Nature remains neutral with regard to jurisdictional claims in published maps and institutional affiliations.

Supplementary Information accompanies this paper at (https://doi.org/ 10.1038/s41398-020-0770-5).
Received: 25 October 2019 Revised: 19 February 2020 Accepted: 24 February 2020

Published online: 13 March 2020

\section{References}

1. Yehuda, R., Giller, E. L., Southwick, S. M., Lowy, M. T. \& Mason, J. W. Hypothalamic-pituitary-adrenal dysfunction in posttraumatic stress disorder. Biol. Psychiatry 30, 1031-1048 (1991).

2. Yehuda, R. Post-traumatic stress disorder. N. Engl. J. Med. 346, 108-114 (2002).

3. Yehuda, R., Golier, J. A., Halligan, S. L., Meaney, M. \& Bierer, L. M. The ACTH response to dexamethasone in PTSD. Am. J. Psychiatry 161, 1397-1403 (2004)

4. Yehuda, R., Boisoneau, D., Lowy, M. T. \& Giller, E. L. Dose-response changes in plasma cortisol and lymphocyte glucocorticoid receptors following dexamethasone administration in combat veterans with and without posttraumatic stress disorder. Arch. Gen. Psychiatry 52, 583-593 (1995).

5. Kessler, R. C., Sonnega, A., Bromet, E., Hughes, M. \& Nelson, C. B. Posttraumatic stress disorder in the National Comorbidity Survey. Arch. Gen. Psychiatry 52, 1048-1060 (1995).

6. Binder, E. B. et al. Association of FKBP5 polymorphisms and childhood abuse with risk of posttraumatic stress disorder symptoms in adults. JAMA 299, 1291-1305 (2008).

7. Binder, E. B. The role of FKBP5, a co-chaperone of the glucocorticoid receptor in the pathogenesis and therapy of affective and anxiety disorders. Psychoneuroendocrinology 34, S186-S195 (2009).

8. Pape, J. C. \& Binder, E. B. The role of genetics and epigenetics in the pathogenesis of posttraumatic stress disorder. Psychiatr. Ann. 46, 510-518 (2016).

9. Wochnik, G. M. et al. FK506-binding proteins 51 and 52 differentially regulate dynein interaction and nuclear translocation of the glucocorticoid receptor in mammalian cells. J. Biol. Chem. 280, 4609-4616 (2005).

10. Hariri, A. R. \& Weinberger, D. R. Imaging genomics. Br. Med. Bull. 65, 259-270 (2003).

11. Huang, H. et al. Imaging genomics. Pac. Symp. Biocomput. 23, 304-306 (2018).

12. Thompson, P. M., Martin, N. G. \& Wright, M. J. Imaging genomics. Curr. Opin. Neurol. 23, 368-373. (2010).

13. Glahn, D. C., Paus, T. \& Thompson, P. M. Imaging genomics: mapping the influence of genetics on brain structure and function. Hum. Brain Mapp. 28 461-463 (2007).

14. Fani, N. et al. Structural and functional connectivity in posttraumatic stress disorder: associations with Fkbp5. Depress Anxiety 33, 300-307 (2016).

15. Roy, A., Gorodetsky, E., Yuan, Q., Goldman, D. \& Enoch, M. A. Interaction of FKBP5, a stress-related gene, with childhood trauma increases the risk for attempting suicide. Neuropsychopharmacology 35, 1674-1683 (2010).

16. Bevilacqua, L. et al. Interaction between FKBP5 and childhood trauma and risk of aggressive behavior. Arch. Gen. Psychiatry 69, 62-70 (2012).

17. Bryant, R. A., Felmingham, K. L., Liddell, B., Das, P. \& Malhi, G. S. Association of FKBP5 polymorphisms and resting-state activity in a frontotemporal-parietal network. Transl. Psychiatry 6, e925 (2016).

18. Morey, R. A. et al. Genome-wide association study of subcortical brain volume in PTSD cases and trauma-exposed controls. Transl. Psychiatry 7, 1265 (2017).

19. Malinen, S. et al. Aberrant temporal and spatial brain activity during rest in patients with chronic pain. Proc. Natl Acad. Sci. USA 107, 6493-6497 (2010).

20. Zang, Y. F. et al. Altered baseline brain activity in children with ADHD revealed by resting-state functional MRI. Brain Dev. 29, 83-91 (2007).

21. Han, Y. et al. Frequency-dependent changes in the amplitude of lowfrequency fluctuations in amnestic mild cognitive impairment: a resting-state fMRI study. Neuroimage 55, 287-295 (2011).

22. Zuo, X. N. et al. The oscillating brain: complex and reliable. Neuroimage 49, 1432-1445 (2010)

23. Bai, F. et al. Association of a GSK-3 $\beta$ polymorphism with brain resting-state function in amnestic-type mild cognitive impairment. J. Alzheimers Dis. 32, 387-396 (2012)

24. Liu, X. et al. The association between TOMM40 gene polymorphism and spontaneous brain activity in amnestic mild cognitive impairment. J. Neurol. 261, 1499-1507 (2014).

25. Cui, L. et al. Relationship between the LHPP gene polymorphism and restingstate brain activity in major depressive disorder. Neural Plast. 2016, 9162590 (2016).

26. Hesketh, T., Lu, L. \& Xing, Z. W. The effect of China's one-child family policy after 25 years. N. Engl. J. Med. 353, 1171-1176 (2005). 
27. Basten, S. \& Jiang, Q. China's family planning policies: recent reforms and future prospects. Stud. Fam. Plann. 45, 493-509 (2014).

28. First, M. B., Spitzer, R. L., Gibbon, M. \& Williams, J. B. Structured Clinical Interview for DSM-IV-TR Axis I Disorders, Research Version, Patient Edition. (SCID-I/P). (Biometrics Research, New York State Psychiatric Institute, New York, 2002).

29. Hamilton, M. A rating scale for depression. J. Neurol. Neurosurg. Psychiatry 23 56 (1960)

30. Hamilton, M. The assessment of anxiety states by rating. Br. J. Med. Psychol. 32, 50-55 (1959).

31. Folstein, M. F., Robins, L. N. \& Helzer, J. E. The mini-mental state examination. Arch. Gen. Psychiatry 40, 812 (1983).

32. Cheng, Y. et al. Social support plays a role in depression in Parkinson's disease: a cross-section study in a Chinese cohort. Parkinsonism Relat. Disord. 14, 43-45 (2008).

33. Jiang, X.-R., Du, J.-J. \& Dong, R.-Y. Coping style, job burnout and mental health of university teachers of the millennial generation. J. Math. Sci. Technol. Educ. 13, 3379-3392 (2017)

34. Ashburner, J. A fast diffeomorphic image registration algorithm. Neuroimage 38, 95-113 (2007).

35. Friston, K. J., Williams, S., Howard, R., Frackowiak, R. S. \& Turner, R. Movementrelated effects in fMRI time-series. Magn. Reson. Med. 35, 346-355 (1996)

36. Yan, C. G. et al. A comprehensive assessment of regional variation in the impact of head micromovements on functional connectomics. Neuroimage 76, 183-201 (2013).

37. Yan, C. G. et al. Reduced default mode network functional connectivity in patients with recurrent major depressive disorder. Proc. Natl Acad. Sci. USA 116, 9078-9083 (2019).

38. Wang, L. et al. Frequency-dependent changes in amplitude of low-frequency oscillations in depression: a resting-state fMRI study. Neurosci. Lett. 614, 105-111 (2016).

39. Wang, Z. et al. Frequency-dependent amplitude alterations of resting-state spontaneous fluctuations in idiopathic generalized epilepsy. Epilepsy Res. 108, 853-860 (2014).

40. Liu, Y. et al. A rapid improved multiplex ligation detection reaction method for the identification of gene mutations in hereditary hearing loss. PLOS ONE 14 e0215212 (2019).

41. Altshuler, D., Donnelly, P. \& Consortium, I. H. A haplotype map of the human genome. Nature 437, 1299 (2005).

42. Barrett, J. C., Fry, B., Maller, J. \& Daly, M. J. Haploview: analysis and visualization of LD and haplotype maps. Bioinformatics 21, 263-265 (2005).

43. Stephens, M., Smith, N. J. \& Donnelly, P. A new statistical method for haplotype reconstruction from population data. Am. J. Hum. Genet. 68, 978-989 (2001)

44. Stephens, M. \& Donnelly, P. A comparison of bayesian methods for haplotype reconstruction from population genotype data. Am. J. Hum. Genet. 73, 1162-1169 (2003).

45. Stephens, M. \& Scheet, P. Accounting for decay of linkage disequilibrium in haplotype inference and missing-data imputation. Am. J. Hum. Genet. 76, 449-462 (2005).

46. Hoehe, M. R. et al. Multiple haplotype-resolved genomes reveal population patterns of gene and protein diplotypes. Nat. Commun. 5, 5569 (2014).

47. Lingjun, Z., Kesheng, W. \& Xingguang, L. Use of diplotypes-matched haplotype pairs from homologous chromosomes-in gene-disease association studies. Shanghai Arch. Psychiatry 26, 165 (2014).

48. Worsley, K. J., Taylor, J. E., Tomaiuolo, F. \& Lerch, J. Unified univariate and multivariate random field theory. Neuroimage 23, S189-S195 (2004).

49. Etkin, A. \& Wager, T. D. Functional neuroimaging of anxiety: a meta-analysis of emotional processing in PTSD, social anxiety disorder, and specific phobia. Am. J. Psychiatry 164, 1476-1488 (2007).
50. Shin, L. M. et al. A functional magnetic resonance imaging study of amygdala and medial prefrontal cortex responses to overtly presented fearful faces in posttraumatic stress disorder. Arch. Gen. Psychiatry 62, 273-281 (2005).

51. Williams, L. M. et al. Trauma modulates amygdala and medial prefrontal responses to consciously attended fear. Neuroimage 29, 347-357 (2006).

52. Boccia, M. et al. Different neural modifications underpin PTSD after different traumatic events: an fMRI meta-analytic study. Brain Imaging Behav. 10, 226-237 (2016).

53. Hayes, J. P., VanElzakker, M. B. \& Shin, L. M. Emotion and cognition interactions in PTSD: a review of neurocognitive and neuroimaging studies. Front. Integr. Neurosci. 6, 89 (2012).

54. Morey, R. A., Petty, C. M., Cooper, D. A., Labar, K. S. \& McCarthy, G. Neural systems for executive and emotional processing are modulated by symptoms of posttraumatic stress disorder in Iraq War veterans. Psychiatry Res. 162, 59-72 (2008).

55. Seeley, W. W. et al. Dissociable intrinsic connectivity networks for salience processing and executive control. J. Neurosci. 27, 2349-2356 (2007).

56. Cabeza, R., Ciaramelli, E. \& Moscovitch, M. Cognitive contributions of the ventral parietal cortex: an integrative theoretical account. Trends Cogn. Sci. 16, 338-352 (2012).

57. Philip, N. S. et al. Regional homogeneity and resting state functional connectivity: associations with exposure to early life stress. Psychiatry Res. 214, 247-253 (2013).

58. Pechtel, P. \& Pizzagalli, D. A. Effects of early life stress on cognitive and affective function: an integrated review of human literature. Psychopharmacology 214, 55-70 (2011).

59. Aupperle, R. L. et al. Dorsolateral prefrontal cortex activation during emotional anticipation and neuropsychological performance in posttraumatic stress disorder. Arch. Gen. Psychiatry 69, 360-371 (2012).

60. Hampshire, A., Chamberlain, S. R., Monti, M. M., Duncan, J. \& Owen, A. M. The role of the right inferior frontal gyrus: inhibition and attentional control. Neuroimage 50, 1313-1319 (2010).

61. Swick, D., Ashley, V. \& Turken, U. Left inferior frontal gyrus is critical for response inhibition. BMC Neurosci. 9, 102 (2008).

62. van Rooij, S. J. et al. Impaired right inferior frontal gyrus response to contextual cues in male veterans with PTSD during response inhibition. J. Psychiatry Neurosci. 39, 330-338 (2014).

63. Stein, M. B., Jang, K. L., Taylor, S., Vernon, P. A. \& Livesley, W. J. Genetic and environmental influences on trauma exposure and posttraumatic stress disorder symptoms: a twin study. Am. J. Psychiatry 159, 1675-1681 (2002).

64. Sartor, C. E. et al. Common heritable contributions to low-risk trauma, high-risk trauma, posttraumatic stress disorder, and major depression. Arch. Gen. Psychiatry 69, 293-299 (2012).

65. Afifi, T. O., Asmundson, G. J., Taylor, S. \& Jang, K. L. The role of genes and environment on trauma exposure and posttraumatic stress disorder symptoms: a review of twin studies. Clin. Psychol. Rev. 30, 101-112 (2010).

66. Hawn, S. E. et al. GxE effects of FKBP5 and traumatic life events on PTSD: a meta-analysis. J. Affect. Disord. 243, 455-462 (2019).

67. Fani, N. et al. FKBP5 and attention bias for threat: associations with hippocampal function and shape. JAMA Psychiatry 70, 392-400 (2013).

68. Tamman, A. J. F. et al. Attachment style moderates effects of FKBP5 polymorphisms and childhood abuse on post-traumatic stress symptoms: Results from the National Health and Resilience in Veterans Study. World J. Biol. Psychiatry 20, 289-300 (2019).

69. Border, R. et al. No support for historical candidate gene or candidate geneby-interaction hypotheses for major depression across multiple large samples. Am. J. Psychiatry 176, 376-387 (2019).

70. Logue, M. W. et al. The Psychiatric Genomics Consortium Posttraumatic Stress Disorder Workgroup: posttraumatic stress disorder enters the age of large-scale genomic collaboration. Neuropsychopharmacology 40, 2287 (2015) 